\title{
The Initial Value Problem for Cubic Semilinear Schrödinger Equations
}

By

\author{
Hiroyuki CHIHARA*
}

\begin{abstract}
We present local and global existence theorems for cubic semilinear Schrödinger equations. Our new results are the improvement of our previous ones ([2], [3], [4]). The idea of the proof consists of the energy and the decay estimates. These equations do not allow the classical energy estimates. To avoid this difficulty, we make strong use of S. Doi's method for linear Schrödinger type equations. Combining cubic nonlinearity and S. Doi's method, we obtain the improved results.
\end{abstract}

\section{\$1. Introduction}

This paper is concerned with the initial value problem for semilinear Schrödinger equations of the form

$$
\begin{gathered}
\partial_{t} u-i \Delta u=F(u, \nabla u) \quad \text { in }(0, \infty) \times \mathbf{R}^{N}, \\
u(0, x)=u_{0}(x) \quad \text { in } \quad \mathbf{R}^{N},
\end{gathered}
$$

where $u(t, x)$ is $\mathbf{C}$-valued, $i=\sqrt{-1}, \quad \partial_{t}=\partial / \partial t, \partial_{j}=\partial / \partial x_{j}, j=1, \cdots, N, \nabla=\left(\partial_{1}\right.$, $\left.\cdots, \partial_{N}\right), \Delta=\partial_{1}^{2}+\cdots+\partial_{N}^{2}$ and $N \in \mathbf{N}$ is the space dimension. We assume that the nonlinear term $F(u, q) \in C^{\infty}\left(\mathbf{R}^{2} \times \mathbf{R}^{2 N} ; \mathbf{C}\right)$ satisfies

$$
|F(u, q)| \leq C\left(|u|^{p}+|q|^{p}\right) \text { near }(u, q)=0
$$

with some integer $p \geq 2$, where $q \in \mathbf{C}^{N}$ corresponds to $\nabla u$. Throughout this paper we use the following notations.

Communicated by T. Kawai, August 16, 1995. Revised November 13.

1991 Mathematics Subject Classifications: 35G25, 35Q55

*Department of Mathematical Sciences, Shinshu University, Matsumoto 390, JAPAN. 


$$
\begin{aligned}
& \frac{\partial}{\partial u}=\frac{1}{2}\left(\frac{\partial}{\partial v_{0}}-i \frac{\partial}{\partial w_{0}}\right), \quad \frac{\partial}{\partial \bar{u}}=\frac{1}{2}\left(\frac{\partial}{\partial v_{0}}+i \frac{\partial}{\partial w_{0}}\right), \\
& \frac{\partial}{\partial q_{j}}=\frac{1}{2}\left(\frac{\partial}{\partial v_{j}}-i \frac{\partial}{\partial w_{j}}\right), \quad \frac{\partial}{\partial \bar{q}_{j}}=\frac{1}{2}\left(\frac{\partial}{\partial v_{j}}+i \frac{\partial}{\partial w_{j}}\right), \\
& v_{0}=\operatorname{Re} u, w_{0}=\operatorname{Im} u, \quad v_{j}=\operatorname{Re} q_{j}, w_{j}=\operatorname{Im} q_{j}, \quad j=1, \cdots, N \text {. }
\end{aligned}
$$

$\mathbb{N}=\{1,2,3, \cdots\}, \mathbb{Z}_{+}=\{0,1,2, \cdots\} . \quad D_{j}=-i \partial_{j}, D=\left(D_{1}, \cdots, D_{N}\right)=-i \nabla, \partial_{\xi_{j}}=$ $\partial / \partial \xi_{j}, \partial_{\xi}=\left(\partial_{\xi_{1}}, \cdots, \partial_{\xi N}\right)$, for $j=1, \cdots, N$.

$$
J_{k} u=e^{\imath \theta\left(t, x_{k}\right)} 2 i(1+t) \partial_{k}\left(e^{-\imath \theta\left(t, x_{k}\right)} u\right)=\left(x_{k}+2 i(1+t) \partial_{k}\right) u
$$

for $k=1, \cdots, N$, where $\theta\left(t, x_{k}\right)=x_{k}^{2} / 4(1+t) . \quad J=\left(J_{1}, \cdots, J_{N}\right) . \quad x^{\alpha}=x_{1}^{\alpha_{1} \cdots} x_{N}^{\alpha_{N}}$,

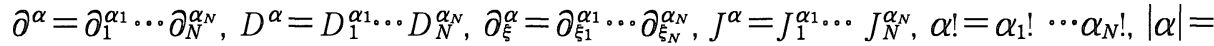
$\alpha_{1}+\cdots+\alpha_{N}$, for any multi index $\alpha=\left(\alpha_{1}, \cdots, \alpha_{N}\right) \in\left(\mathbb{Z}_{+}\right)^{N} .\langle\xi\rangle=\sqrt{1+|\xi|^{2}},\left\langle\xi_{j}\right\rangle$ $=\sqrt{1+\xi_{j}^{2}},\langle D\rangle=(1-\Delta)^{1 / 2},\left\langle D_{j}\right\rangle=\left(1-\partial_{j}^{2}\right)^{1 / 2}$.

$$
W^{s, r}=W^{s, r}\left(\mathbb{R}^{N}\right)=\left\{u \in \mathscr{S}^{\prime}\left(\mathbb{R}^{N}\right) \mid\|u\|_{W} s, r=\left(\int_{\mathbb{R}^{N}}\left|\langle D\rangle^{s} u\right|^{r} d x\right)^{1 / r}<+\infty\right\},
$$

$L^{r}=W^{0, r}, H^{s}=W^{s, 2}$ for $s \in \mathbb{R}$ and $1 \leq r<\infty$.

$$
W^{s, \infty}=W^{s, \infty}\left(\mathbb{R}^{N}\right)=\left\{u \in \mathscr{S}^{\prime}\left(\mathbb{R}^{N}\right) \mid\|u\|_{W} s, \infty=\text { ess. } \sup \left|\langle D\rangle^{s} u\right|<+\infty\right\},
$$

$L^{\infty}=W^{0, \infty}$, for $s \in \mathbb{R}$.

$$
H^{s, s^{\prime}}=H^{s, s^{\prime}}\left(\mathbb{R}^{N}\right)=\left\{u \in \mathscr{S}^{\prime}\left(\mathbb{R}^{N}\right) \mid\left\|\langle x\rangle^{s^{\prime}}\langle D\rangle^{s} u\right\|_{L^{2}}<+\infty\right\}
$$

for $s, s^{\prime} \in \mathbb{R} .\|\bullet\|_{s}$ means $\left(H^{s}\right)^{2}$-norm. Especially $\|\cdot\|$ and $(\cdot, \cdot)$ mean $\left(L^{2}\right)^{2}$-norm and $\left(L^{2}\right)^{2}$-inner product respectively. $\quad \&=\mathscr{\&}\left(\mathbb{R}^{N}\right)$ and $\mathscr{S}^{\prime}=\mathscr{S}^{\prime}\left(\mathbb{R}^{N}\right)$ denote the Schwartz class and its topological dual space respectively. $\mathscr{B}^{\infty}=\mathscr{B}^{\infty}\left(\mathbb{R}^{N}\right)$ is the set of all $C^{\infty}$-functions on $\mathbb{R}^{N}$ whose derivatives of any order are all bounded. When $X$ and $Y$ are normed vector spaces, $\mathscr{L}(X, Y)$ and $\mathscr{L}(X)$ are the set of all bounded linear operators of $X$ to $Y$ and of $X$ to $X$ respectively. $\check{x}_{j}=\left(x_{1}, \cdots, x_{j-1}\right.$, $\left.x_{j+1}, \cdots, x_{N}\right)$, for $x \in \mathbb{R}^{N}$ and $j=1, \cdots, N . \quad \delta_{j k}$ is Kronecker's delta, i.e., $\delta_{j k}=1$ if $j$ $=k, \delta_{j k}=0$ otherwise. $[s]$ means the largest integer less than or equal to $s \geq 0$. Different positive constants might be denoted by the same letter $C$.

In this problem the difficulty of so-called loss of derivatives takes place because the nonlinear term $F(u, \nabla u)$ contains $\nabla u$. More precisely, if 


$$
\operatorname{Im} \frac{\partial F}{\partial q_{j}}(u, q) \equiv 0, \quad j=1, \cdots, N
$$

then one can obtain the classical energy estimates for the solution $u$. In general the classical energy estimates do not hold without (1.3). Then studies on the initial value problem (1.1)-(1.2) have been mainly concerned with the case of (1.3). Recently, however, several researchers have been studying (1.1)-(1.2) without the condition (1.3) ([1], [2], [3], [4], [7], [8], [10], [11], [18], [20]). Except for [11], these works are the application of the theory of linear Schrödinger type equations (see S. Mizohata [14, Lecture VII] and S. Doi [5] for instance). Especially S. Doi's method is effective for the application to semilinear equations. In fact, using his method, the author studied the local and the global existence for general semilinear equations which do not satisfy (1.3) (see [2], [3] and [4]). On the other hand, C. E. Kenig-G. Ponce-L. Vega ([11]) obtained the sharp version of the smoothing property of $e^{\imath t \Delta}$ and applied it to the local existence of small solutions to the general semilinear equations.

The purpose of this paper is to improve the results in [2], [3] and [4]. If the nonlinear term $F(u, q)$ is cubic $(p \geq 3)$, then the Taylor formula gives

$$
F(u, q)=F_{3}(u, q)+F_{4}(u, q) \text { near }(u, q)=0
$$

where $F_{3}(u, q)$ is a cubic homogeneous polynominal of $u, \bar{u}, q, \bar{q}$ and $F_{4}(u, q)$ is a higher order term. We shall prove

Theorem 1.1. We assume $N \geq 2$ and $p \geq 3$. Then there exists a sufficently large integer $m_{1} \in \mathbb{N}$ such that for any $u_{0} \in H^{m}\left(m \geq m_{1}\right)$, there exists a time $T$ $=T\left(\left\|u_{0}\right\|_{H^{m_{1}}}\right)>0$ such that the initial value problem (1.1)-(1.2) rossesses a $u$ nique solution $u \in C\left([0, T) ; H^{m}\right)$.

Theorem 1.2. We assume $N \geq 3$ and $p \geq 3$. Then there exist sufficiently large integer $m_{2} \in \mathbb{N}$ and a small constant $\delta>0$ such that for any

$$
u_{0} \in \bigcap_{j=0}^{2} H^{m-2 j, j}\left(m \geq m_{2}+2\right) \text { satisfying } \sum_{j=0}^{2}\left\|u_{0}\right\|_{H^{m_{2}-2 j, j}} \leq \delta,
$$

the initial value problem (1.1)-(1.2) possesses a unique solution

$$
u \in \bigcap_{j=0}^{2} C\left([0, \infty) ; H^{m-2 j, j}\right)
$$

and 
Theorem 1.3. We assume $N=2, p \geq 3$ and $F_{3}(u, q)$ satisfies the gauge invariance

$$
F_{3}\left(e^{i \theta} u, e^{i \theta} q\right)=e^{i \theta} F_{3}(u, q) \quad \text { for } \quad(u, q) \in \mathbb{C} \times \mathbb{C}^{N}, \quad \theta \in \mathbb{R} .
$$

Then there exist a sufficiently large integer $m_{3} \in \mathbb{N}$ and a small constant $\delta>0$ such that for any

$$
u_{0} \in \bigcap_{j=0}^{1} H^{m-2 j, j} \quad\left(m \geq m_{3}+2\right) \text { satisfying } \sum_{j=0}^{1}\left\|u_{0}\right\|_{H^{m_{3}-2 \jmath, j}} \leq \delta
$$

the initial value problem (1.1)-(1.2) possesses a unique solution

$$
u \in \bigcap_{j=0}^{1} C\left([0, \infty) ; H^{m-2 j, j}\right)
$$

Remark 1.1. Because we use pseudo-differential operators, it is troublesome to determine the minimum value of $m_{1}, m_{2}$ and $m_{3}$.

Remark 1.2. In [2] the author proved the similar results for the case of $p \geq 2$. There we made use of the weighted Sobolev space $H^{m} \cap H^{m-1,1}$ for $p \geq$ 3 or $H^{m} \cap H^{m-2,2}$ for $p \geq 2$. Hence Theorem 1.1 is an improvement of [2] in the case of $p \geq 3$.

Remark 1.3. In [3] and [4] the author studied the global existence for general semilinear equations: if we assume

$$
N(p-1)^{2} / 2 p>\left\{\begin{array}{ll}
2, & p \geq 3 \\
3, & p=2
\end{array}\right. \text {, }
$$

or if we assume the gauge invariance

$$
F\left(e^{i \theta} u, e^{i \theta} q\right)=e^{i \theta} F(u, q) \text { for }(u, q) \in \mathbb{C} \times \mathbb{C}^{N}, \quad \theta \in \mathbb{R},
$$

and $N(p-1) / 2>2$, then the global existence results hold. Clearly Theorems 1.2 and 1.3 are improvement of [3] and [4]. This is basically due to the device for how to apply S. Doi's method to semilinear equations. Unfortunately this device is not applicable to quadratic nonlinearity. We explain the detail at 
the end of $\S 2$ (Remark 2.1).

Now we give the strategy of the proof. Theorem 1.1 is proved by the parabolic regularization and the uniform estimates which follow from the energy estimates. Theorems 1.2 and 1.3 are proved by Theorem 1.1 and a priori estimates which consists of the energy and the decay estimates.

For the energy estimates, we see the equation (1.1) as a system of $(u, \bar{u})$ because the nonlinear term $F(u, \nabla u)$ contains not only $\nabla u$ but also $\nabla \bar{u}$. After the diagonalization of this system modulo bounded operators, this system becomes a couple of single Schrödinger type equation essentially. Then we can use S. Doi's method and obtain the energy inequality.

On the other hand, we get the decay estimates by the operator $J$ and the Gagliardo-Nirenberg inequality (see Lemma 3.1). It is well-known that this technique is effective for the case of (1.5) because $J$ acts on the nonlinear term $F(u, \nabla u)$ satisfying (1.5) as if it were the usual differentiation $\partial$. Conversely $J$ does not act well without (1.5): the more we use $J$, the more the loss of decay becomes. N. Hayashi ([6]) made good use of $J$ for some quadratic nonlinear term satisfying (1.3) and obtained some extension of [13] and [19]. We also use the similar technique to that of [6].

The organization of this paper is as follows. $\$ 2$ consists of the linear estimates for some Schrödinger type systems. $\S 3$ contains preliminary results. In $\S 4, \S 5$ and $\S 6$ we prove Theorems $1.1,1.2$ and 1.3 respectively.

\section{§2. Linear Estimates}

In this section we study the initial value problem for the following linear Schrödinger type systems

$$
\begin{array}{cl}
L v \equiv\left(I \partial_{t}+i H(t)\right) v=f(t, x) & \text { in }(0, T) \times \mathbb{R}^{N} \\
v(0, x)=v_{0}(x) & \text { in } \mathbf{R}^{N},
\end{array}
$$

where $v=v(t, x)$ is $\mathbb{C}^{2}$-valued, $I$ is the $2 \times 2$ identity matrix and the operator $H(t)=h(t, x, D)$ is defined by

$$
\begin{aligned}
& h(t, x, \xi)=a(\xi)+b(t, x, \xi), \quad a(\xi)=\left[\begin{array}{cc}
|\xi|^{2} & 0 \\
0 & -|\xi|^{2}
\end{array}\right], \\
& b(t, x, \xi)=\left[\begin{array}{ll}
b_{11}(t, x, \xi) & b_{12}(t, x, \xi) \\
b_{21}(t, x, \xi) & b_{22}(t, x, \xi)
\end{array}\right]=\sum_{j=1}^{N}\left[\begin{array}{ll}
b_{11 j}(t, x) & b_{12 j}(t, x) \\
b_{21 j}(t, x) & b_{22 j}(t, x)
\end{array}\right] \xi_{j}, \\
& b_{m n j}(t, x) \in C^{1}\left([0, T] ; \mathscr{B}^{\infty}\right), \quad m, n=1,2 \text { and } j=1, \cdots, N .
\end{aligned}
$$


For the convenience, we put

$$
b^{\text {diag }}(t, x, \xi)\left[\begin{array}{cc}
b_{11}(t, x, \xi) & 0 \\
0 & b_{22}(t, x, \xi)
\end{array}\right], \quad b^{\text {off }}(t, x, \xi)=\left[\begin{array}{cc}
0 & b_{12}(t, x, \xi) \\
b_{21}(t, x, \xi) & 0
\end{array}\right]
$$

When we apply the linear estimates in this section to (1.1)-(1.2), we see $v$ as $v$ $={ }^{t}(u, \bar{u})$. For $H^{s}$-wellposedness of $(2.1)-(2.2)$, we assume the Doi type condition on $b^{\text {diag }}(t, x, \xi)$, that is to say, there exist functions

$$
\phi_{j}(t, s) \in C\left([0, T] ; \mathscr{B}^{\infty}(\mathbb{R})\right) \cap C^{1}\left([0, T] ; L^{1}(\mathbb{R})\right), \quad j=1, \cdots, N
$$

such that

$$
\left|\operatorname{Im} b_{n n j}(t, x)\right| \leq \phi_{j}\left(t, x_{j}\right) \quad \text { for } \quad(t, x) \in[0, T] \times \mathbb{R}^{N}, \quad n=1,2, j=1, \cdots, N .
$$

Our analysis is based on the symbolic calculus for pseudo-differential operators (see [9] or [14] for instance).

For $H^{s_{-}}$-wellposedness we have

Proposition 2.1. We assume (2.3). Then the initial value problem (2.1) (2.2) is $H^{s}$-wellposed for any $s \in \mathbb{R}$, that is to say, for any $v_{0}(x) \in\left(H^{s}\right)^{2}$ and for any $f(t, x) \in\left(L_{\mathrm{loc}}^{1}\left(0, T ; H^{s}\right)\right)^{2},(2.1)-(2.2)$ possesses a unique solution $v \in$ $\left(C\left([0, T] ; H^{s}\right)\right)^{2}$.

We have only prove Proposition 2.2 with $s=0$. Our strategy divides into two steps. At the first step we diagonalize the operator $H(t)$ modulo bounded operators. Roughly speaking, its symbol

$$
h(t, x, \xi)=\left[\begin{array}{cc}
|\xi|^{2}+b_{11}(t, x, \xi) & b_{12}(t, x, \xi) \\
b_{21}(t, x, \xi) & -|\xi|^{2}+b_{22}(t, x, \xi)
\end{array}\right]
$$

has two distinct eigen-values provided that $|\xi|$ is sufficiently large. Thus we can easily diagonalize $h(t, x, \xi)$ and therefore this system becomes a couple of single Schrödinger type equations essentially. At the second step we apply S. Doi's method ([5]) to the diagonalized system.

Following [2] or [3], we explain the outline of proof of Proposition 2.2. We introduce some pseudo-differential operators for this purpose. The diagonalization is carried out by 


$$
\begin{gathered}
\Lambda(t)=I+\tilde{\Lambda}(t), \quad \Lambda^{\prime}(t)=I-\tilde{\Lambda}(t), \quad \tilde{\Lambda}(t)=\tilde{\lambda}(t, x, D), \\
\tilde{\lambda}(t, x, \xi)=\frac{1}{2} \sum_{j=1}^{N}\left[\begin{array}{cc}
0 & b_{12}(t, x) \\
-b_{21 j}(t, x) & 0
\end{array}\right] \xi_{j}\langle\xi\rangle^{-2} .
\end{gathered}
$$

The loss of derivatives is resolved by

$$
\begin{array}{rlrl}
K(t) & =k(t, x, D), & K^{\prime}(t) & =k^{\prime}(t, x, D), \\
k(t, x, \xi) & =\left[\begin{array}{cc}
k_{1}(t, x, \xi) & 0 \\
0 & k_{1}^{\prime}(t, x, \xi)
\end{array}\right], & k^{\prime}(t, x, \xi) & =\left[\begin{array}{cc}
k_{1}^{\prime}(t, x, \xi) & 0 \\
0 & k_{1}(t, x, \xi)
\end{array}\right], \\
k_{1}(t, x, \xi) & =\exp (-p(t, x, \xi)), & k_{1}^{\prime}(t, x, \xi) & =\exp (p(t, x, \xi)), \\
p(t, x, \xi) & =\sum_{j=1}^{N} \int_{0}^{x} \phi_{j}(t, s) d s \xi_{j}\left\langle\xi_{j}\right\rangle^{-1} . &
\end{array}
$$

It is convenient to use the following notations

$$
\begin{aligned}
& B_{K}(t)=\sup _{(x, \xi) \in \mathbf{R}^{N} \times \mathbf{R}^{N}} \sum_{|\alpha+\beta| \leq l}\left|\partial_{\xi}^{\alpha} D^{\beta} k_{1}(t, x, \xi)\right|+\sup _{(x, \xi) \in \mathbf{R}^{N} \times \mathbf{R}^{N}} \sum_{|\alpha+\beta| \leq l}\left|\partial_{\xi}^{\alpha} D^{\beta} k_{1}(t, x, \xi)^{-1}\right|, \\
& B_{b}(t)=\sum_{m, n=1,2} \sum_{j=1}^{N} \sum_{|\alpha| \leq l}\left(\sup _{x \in \mathbf{R}^{N}}\left|\partial^{\alpha} b_{m n j}(t, x)\right|+\sup _{x \in \mathbf{R}^{N}}\left|\partial_{t} \partial^{\alpha} b_{m n j}(t, x)\right|\right) \\
& B_{\phi}^{0}(t)=\sum_{j=1}^{N} \int_{-\infty}^{+\infty} \phi_{j}\left(t, x_{j}\right) d x_{j}, \quad B_{\phi}^{1}(t)=\sum_{j=1}^{N} \sup _{x, \in \mathbf{R}}\left|\int_{0}^{x} \partial_{t} \phi_{j}\left(t, y_{j}\right) d y_{j}\right|, \\
& B_{\phi}^{\infty}(t)=\sum_{j=1}^{N} \sum_{k=0}^{l} \sup _{x, \in \mathbf{R}}\left|\partial_{j}^{k} \phi_{j}\left(t, x_{j}\right)\right|
\end{aligned}
$$

where $l \in \mathbf{N}$ is large enough to be used in this section. It is very troublesome to find the minimum value of $l . m_{1}, m_{2}$ and $m_{3}$ in $\S 1$ is determined by $N$ and $l$ (see Remark 1.1). To eliminate the loss of derivatives in (2.1), we use the map $v$ $\mapsto K(t) \Lambda(t) v$. Before we use this map, we verify that this is automorphic on $(L)^{2}$ in some sense.

Lemma 2.2. There exists a constant $C>0$ such that

$$
\begin{aligned}
\|v\| & \leq C B_{K}(t)\left(1+B_{b}(t)\right)^{2}\left(1+B_{\phi}^{0}(t) B_{\phi}^{\infty}(t)\right) N_{t}(v), \\
N_{t}(v) & \leq C B_{K}(t)\left(1+B_{b}(t)\right)\|v\|
\end{aligned}
$$

for $v \in\left(L^{2}\right)^{2}$ and $t \in[0, T]$, where $N_{t}(v)=\|K(t) \Lambda(t) v\|+\|v\|_{-1}$. 
Proof. See [2] or [3].

Using $K(t), K^{\prime}(t), \Lambda(t)$ and $\Lambda^{\prime}(t)$, we get

$$
\begin{aligned}
& K(t) \Lambda(t) L v=\left(I \partial_{t}+i a(D)+q(t, x, D)\right) K(t) \Lambda(t) v+R_{1}(t) v, \\
q(t, x, \xi)= & 2 I \sum_{j=1}^{N} \phi_{j}\left(t, x_{j}\right) \xi_{j}\left\langle\xi_{j}\right\rangle^{-1}+i b^{\operatorname{diag}}(t, x, \xi) \\
= & \sum_{j=1}^{N}\left[\begin{array}{cc}
2 \phi_{j}\left(t, x_{j}\right) \xi_{j}\left\langle\xi_{j}\right\rangle^{-1}+i b_{11 j}(t, x) \xi_{j} & 0 \\
0 & 2 \phi_{j}\left(t, x_{j}\right) \xi_{j}\left\langle\xi_{j}\right\rangle^{-1}+i b_{22 j}(t, x) \xi_{j}
\end{array}\right],
\end{aligned}
$$

where $R_{1}(t)$ is a bounded operator which is estimated as

$\left\|R_{10}(t)\right\| \mathscr{L}\left(\left(L^{2}\right)^{2}\right) \leq C\left(B_{b}(t)+B_{\phi}^{1}(t)+B_{\phi}^{\infty}(t)\right)\left(1+B_{\phi}^{\infty}(t)\right)\left(1+B_{b}(t)\right)^{3} B_{K}(t)$.

To complete the proof of Proposition 2.2, we tave only to show the following energy inequalities. More precisely, Proposition 2.2 follows from the duality type arguments for the forward and the backward initial value problems. The energy inequalities play an essential role in these arguments. See L. Hörmander [9, section 23.1] for the detail.

Lemma 2.3. There exists a constant $C_{T}>0$ such that

$$
\begin{aligned}
& \|v(t)\| \leq C_{T}\left(\|v(0)\|+\int_{0}^{t}\|(L v)(\tau)\| d \tau\right), \\
& \|v(t)\| \leq C_{T}\left(\|v(T)\|+\int_{t}^{T}\left\|\left(L^{*} v\right)(\tau)\right\| d \tau\right),
\end{aligned}
$$

for $v \in\left(C\left([0, T] ; H^{2}\right) \cap C^{1}\left([0, T] ; L^{2}\right)^{2}, \quad t \in[0, T]\right.$.

Here $L^{*}$ is the formally adjoint operator of $L$.

Proof. We put $w=K(t) \Lambda(t) v, \quad f(t, x)=(L v)(t, x)$ and $g=K(t) \Lambda(t) f$. Applying (2.3) and the sharp Gårding inequality to (2.6), we get

$$
\begin{aligned}
\frac{d}{d t}\|w(t)\| & \leq C\left(B_{b}(t)+B_{\phi}^{1}(t)+B_{\phi}^{\infty}(t)\right) B_{\text {etc }}(t) N_{t}(v(t))+\|g(t)\|, \\
B_{\text {etc }}(t) & =\left(1+B_{b}(t)\right)^{5}\left(1+B_{\phi}^{0}(t)\right)\left(1+B_{\phi}^{\infty}(t)\right)^{2} B_{K}(t)^{2} .
\end{aligned}
$$

On the other hand, we have 


$$
\frac{d}{d t}\|v(t)\|_{-1} \leq C B_{b}(t) B_{\mathrm{etc}}(t) N_{t}(v(t))+\|f(t)\|_{-1}
$$

Combining (2.10) and (2.11), we obtain

$$
\frac{d}{d t} N_{t}(v(t)) \leq C\left(B_{b}(t)+B_{\phi}^{1}(t)+B_{\phi}^{\infty}(t)\right) B_{\text {etc }}(t) N_{t}(v(t))+N_{t}(f(t))
$$

(2.4), (2.5) and (2.12) implies (2.8). In the same way we can get (2.9). This completes the proof of Lemma 2.3 and then we finish the proof of Proposition 2.1 .

Remark 2.1. When we apply Proposition 2.1 or Lemma 2.3 to (1.1) - (1.2), we take $\phi_{j}\left(t, x_{j}\right)$ satisfying

$$
\sup _{\bar{x}, \in \mathbf{R}^{N-1}}\left|\frac{\partial F}{\partial q_{j}}(u, \nabla u)(t, x)\right| \leq \phi_{j}\left(t, x_{j}\right), \quad j=1, \cdots, N
$$

Then we choose $\phi_{j}\left(t, x_{j}\right)$ as

$$
\phi_{j}\left(t, x_{j}\right)=M \int_{\mathbf{R}^{N-1}}\left|\langle D\rangle^{[(N-1) / 2]+2} u(t, x)\right|^{2} d \bar{x}_{j}
$$

or

$$
\phi_{j}\left(t, x_{j}\right)=M(1+t)^{-d} \int_{\mathbb{R}^{N-1}}\left|\langle D\rangle^{[(N-1) / 2]+1} J u(t, x)\right|^{2} d \bar{x}_{j}
$$

with some constants $M>0$ and $d>0$. On the other hand, in [2], [3] and [4] we chose $\phi_{j}\left(t, x_{j}\right)$ as

$$
\phi_{j}\left(t, x_{j}\right)=\phi\left(x_{j}\right)=M\left\langle x_{j}\right\rangle^{-1-\delta}
$$

or

$$
\phi_{j}\left(t, x_{j}\right)=M(1+t)^{-d}\left\langle x_{j}\right\rangle^{-1-\delta}
$$

with some constants $M>0, \delta>0$ and $d>0$. (2.14) or (2.16) is applied to the local existence. To use (2.16), we need the spatial decay of the solution and then we have to introduce the weighted Sobolev spaces. On the other hand, we make use of (2.15) or (2.17) to obtain the global existence results. (2.17) causes generally loss of time-decay and (2.15) does not. (2.15) has the structural nice pro- 
perty. In [10] S. Katayama Y. Tsutsumi made good use of this property to study the global existence theorem for (1.1)-(1.2) in one space dimension $(N=$ $1)$. In the present paper this is not essential necessarily. It is more important that $\phi_{,}(t, \cdot)$ is smooth enough to be treated by the symbolic calculus. Unfortunately, when $F(u, q)$ is quadratic, (2.14) and (2.15) are not applicable to (1.1) (1.2). It seems to be difficult to find sufficiently smooth functions $\phi_{j}\left(t, x_{j}\right), j=1$, $\cdots, N$, which give no loss of time-decay and are applicable to the quadratic nonlinearity.

\section{§3. Preliminaries}

This section is devoted to the estimates on the nonlinear term $F(u, \nabla u)$. Here the Gagliardo-Nirenberg inequality plays an important role.

\section{Lemma $3_{1} 1 .(T h e$ Gagliardo-Nirenberg inequality)}

Let $r_{0}, r_{1}$, and $r_{2}$ satisfy $1 \leq r_{0}, r_{1}, r_{2} \leq \infty$, and let $j_{1}$ and $j_{2}$ be integers satisfying 0 $\leq j_{1}<j_{2}$. Then there exists a constant $C_{0}=C_{0}\left(N, j_{1}, j_{2}, r_{0}, r_{2}, a\right)>0$ such that for any $u \in L^{r_{0}}$ satisfying $\partial^{\alpha} u \in L^{r_{2}},|\alpha|=j_{2}$, the following inequalities hold

$$
\sum_{|\beta|=j_{1}}\left\|\partial^{\beta} u\right\|_{L^{r_{1}}} \leq C_{0} \sum_{|\alpha|=j_{2}}\left\|\partial^{\alpha} u\right\|_{L^{r_{2}}}^{\alpha}\|u\|_{L^{r_{0}}}^{1-a}
$$

where

$$
\frac{1}{r_{1}}=\frac{j_{1}}{N}+a\left(\frac{1}{r_{2}}-\frac{j_{2}}{N}\right)+(1-a) \frac{1}{r_{0}}
$$

for all $a$ in the interval

$$
\frac{j_{1}}{j_{2}} \leq a \leq 1,
$$

with the following exceptional cases

i ) If $j_{1}=0, r_{2} j_{2}<N, r_{0}=\infty$, then we make the additional assumption that $u(x) \rightarrow$ 0 as $|x| \rightarrow+\infty$.

ii) If $1<r_{2}<\infty$, and $j_{2}-j_{1}-N / r_{2} \in \mathbb{Z}_{+}$, then (3.1) holds only for a satisfying $j_{1} / j_{2} \leq a<1$.

Proof. See L. Nirenberg [17] for instance.

To prove Theorem 1.1 we prepare 
Lemma 3.2. We assume $F(u, q) \in C^{\infty}\left(\mathbb{R}^{2} \times \mathbf{R}^{2 N} ; \mathbf{C}\right)$ and $F(0,0)=0$. Let $m$ be an integer $>N / 2+1$. Then there exists a non-decreasing function $A_{m}(\cdot)$ on $[0, \infty)$ such that for any $u, v \in H^{m}$,

$$
\begin{gathered}
\|F(u, \nabla u)\|_{H^{m-1}} \leq A_{m}\left(\|u\|_{W^{1, \infty}}\right)\|u\|_{H^{m}}, \\
\sum_{|\alpha|=m}\left\|\partial^{\alpha} F(u, \nabla u)-P\left(u, \nabla u, \partial \partial^{\alpha} u\right)\right\|_{L^{2}} \leq A_{m}\left(\|u\|_{W^{1, \infty}}\right)\|u\|_{H^{m}}, \\
\|F(u, \nabla u)-F(v, \nabla v)\|_{H^{m-1}} \leq A_{m}\left(\|u\|_{H^{m}}+\|v\|_{H^{m}}\right)\|u-v\|_{H^{m}},
\end{gathered}
$$

where

$$
P\left(u, \nabla u, \partial \partial^{\alpha} u\right)=\sum_{j=1}^{N} \frac{\partial F}{\partial q_{j}}(u, \nabla u) \partial_{j} \partial^{\alpha} u+\sum_{j=1}^{N} \frac{\partial F}{\partial \bar{q}_{j}}(u, \nabla u) \partial_{j} \partial^{\alpha} \bar{u}
$$

Proof. Making good use of the Gagliardo-Nirenberg inequality we can show Lemma 3.2. This is basically due to J. Morser ([16]) (see also S. Klainer$\operatorname{man}[12])$.

Now we prepare to obtain the estimates on the nonlinear term in order to prove the global existence theorem. Especially we get the decay estimates by the operator $J$ and the Gagliardo-Nirenberg inequality. Here we note the properties of $J$ :

$$
\left[J_{k}, \partial_{t}-i \Delta\right]=\left[J_{k}, J_{l}\right]=0,\left[\partial_{k}, J_{l}\right]=\delta_{k l}, \quad k, l=1, \cdots, N
$$

To get the decay estimates we prepare

\section{Lemma 3.3.}

(1) Let $N=1$. For any $u \in H^{1} \cap H^{0,1}$,

$$
\|u\|_{L^{\infty}} \leq C(1+t)^{-1 / 2}\left\|J_{1} u\right\|_{L^{2}}^{1 / 2}\|u\|_{L^{2}}^{1 / 2} .
$$

(2) Let $N=2$. For any $v \in H^{2} \cap H^{1,1}$,

$$
\|v\|_{L^{\infty}} \leq C(1+t)^{-3 / 4} \sum_{\substack{|\alpha| \leq 1 \\|\beta|=1}}\left\|\partial^{\alpha} J^{\beta} v\right\|_{L^{2}}^{3 / 4}\|v\|_{L^{2}}^{1 / 4}
$$

(3) Let $N=3$. For any $w \in H^{3} \cap H^{1,2}$, 


$$
\begin{aligned}
\|w\|_{L^{\infty}} \leq C(1+t)^{-3 / 2} \sum_{|\beta|=2}\left\|J^{\beta} w\right\|_{L^{2}}^{1 / 2} \sum_{\left|\beta^{\prime}\right|=1}\left\|J^{\beta^{\prime}} w\right\|_{L^{2}}^{1 / 2} \\
\sum_{\left|\beta^{\prime}\right|=1}\left\|J^{\beta^{\prime}} w\right\|_{L^{\infty}} \leq C(1+t)^{-1} \sum_{\substack{|\alpha| \leq 1 \\
|\beta|=2}}\left\|\partial^{\alpha} J^{\beta} w\right\|_{L^{2}}
\end{aligned}
$$

Proof. Let $\theta=\theta(t, x)=\theta\left(t, x_{1}\right)+\cdots+\theta\left(t, x_{N}\right)=|x|^{2} / 4(1+t)$.

To show (3.5), we use (3.1) with $N=1, r_{0}=2, r_{1}=\infty, r_{2}=2, j_{1}=0, j_{2}=1$ and $a=1 / 2$. Then we have

$$
\begin{aligned}
\|u\|_{L^{\infty}} & =\left\|e^{-i \theta} u\right\|_{L^{\infty}} \\
& \leq C\left\|\partial_{1}\left(e^{-i \theta} u\right)\right\|_{L^{2}}^{1 / 2}\left\|e^{-i \theta} u\right\|_{L^{2}}^{1 / 2} \\
& =C\left\|e^{i \theta} \partial_{1}\left(e^{-i \theta} u\right)\right\|_{L^{2}}^{1 / 2}\|u\|_{L^{2}}^{1 / 2} \\
& \leq C(1+t)^{-1 / 2}\left\|J_{1} u\right\|_{L^{2}}^{1 / 2}\|u\|_{L^{2}}^{1 / 2} .
\end{aligned}
$$

Next we show (3.6). By (3.1) with $N=2, r_{0}=4, r_{1}=\infty, r_{2}=4, j_{1}=0, j_{2}=1$ and $a=1 / 2$ we get

$$
\begin{aligned}
\|v\|_{L^{\infty}} & =\left\|e^{-i \theta} v\right\|_{L^{\infty}} \\
& \leq C(1+t)^{-1 / 2} \sum_{|\beta|=1}\left\|J^{\beta} v\right\|_{L^{4}}^{1 / 2}\|v\|_{L^{4}}^{1 / 2}
\end{aligned}
$$

Making use of (3.1) again with $N=2, r_{0}=2, r_{1}=4, r_{2}=2, j_{1}=0, j_{2}=1$ and $a=1 / 2$ we have

$$
\begin{gathered}
\sum_{|\beta|=1}\left\|J^{\beta} v\right\|_{L^{4}} \leq \sum_{\substack{|\alpha|=1 \\
|\beta|=1}}\left\|\partial^{\alpha} J^{\beta} v\right\|_{L^{2}}^{1 / 2} \sum_{\left|\beta^{\prime}\right|=1}\left\|J^{\beta^{\prime}} v\right\|_{L^{2}}^{1 / 2}, \\
\|v\|_{L^{4}} \leq C(1+t)^{-1 / 2} \sum_{|\beta|=1}\left\|J^{\beta} v\right\|_{L^{2}}^{1 / 2}\|v\|_{L^{2}}^{1 / 2} .
\end{gathered}
$$

Combining $(3.9) \sim(3.11)$ we obtain (3.6).

For (3.7), by (3.1) with $N=3, r_{0}=6, r_{1}=\infty, r_{2}=6, j_{1}=0, j_{2}=1$ and $a=1 / 2$ we have

$$
\|w\|_{L^{\infty}} \leq C(1+t)^{-1 / 2} \sum_{|\beta|=1}\left\|J^{\beta} w\right\|_{L^{6}}^{1 / 2}\|v\|_{L^{6}}^{1 / 2}
$$

Making use of (3.1) with $N=3, r_{0}=2, r_{1}=6, r_{2}=2, j_{1}=0, j_{2}=1$ and $a=1$ once again, we can obtain (3.7). Similarly we can show (3.8). 
Under the assumption of Theorems 1.2 and 1.3, we introduce the following notations

$$
\begin{gathered}
X_{m}^{N}(t)= \begin{cases}\sum_{\substack{|\alpha+2 \beta| \leqq m \\
|\beta| \leqq 1}}\left\|\partial^{\alpha} J^{\beta} u(t)\right\|_{L^{2}}+(1+t)^{-1 / 4} \sum_{\substack{|\alpha| \leq m-4 \\
|\beta|=2}}\left\|\partial^{\alpha} J^{\beta} u(t)\right\|_{L^{2},} & \text { if } N \geq 3, \\
\sum_{|\alpha| \leqq m}\left\|\partial^{\alpha} u(t)\right\|_{L^{2}}+\sum_{\substack{|\alpha| \leqq 3 \\
|\beta|=1}}\left\|\partial^{\alpha} J^{\beta} u(t)\right\|_{L^{2},} & \text { if } N=2,\end{cases} \\
\text { where } m \geq \begin{cases}m_{0}=\left[\frac{N+1}{2}\right]+6, & \text { if } N \geq 3, \\
7, & \text { if } N=2,\end{cases}
\end{gathered}
$$$$
P_{\alpha}=P\left(u, \nabla u, \partial \partial^{\alpha} u\right)
$$$$
=\sum_{j=1}^{N} \frac{\partial F}{\partial q_{j}}(u, \nabla u) \partial_{j} \partial^{\alpha} u+\sum_{j=1}^{N} \frac{\partial F}{\partial \bar{q}_{j}}(u, \nabla u) \partial_{j} \partial^{\alpha} \bar{u},
$$$$
P_{\alpha \beta}=P^{\prime}\left(u, \nabla u, \partial \partial^{\alpha} J^{\beta} u\right)
$$

$$
= \begin{cases}\sum_{j=1}^{N} G_{j}(u, \nabla u) \partial_{j} \partial^{\alpha} J^{\beta} u+\sum_{j=1}^{N} G_{j}^{\prime}(u, \nabla u) \overline{\partial_{j}} \overline{\partial^{\alpha} J^{\beta} u,} & \text { if } N \geq 3, \\ \sum_{j=1}^{N} \frac{\partial F_{3}}{\partial q_{j}}(u, \nabla u) \partial_{j} \partial^{\alpha} J^{\beta} u+(-1)^{|\beta|} \sum_{j=1}^{N} \frac{\partial F_{3}}{\partial \bar{q}_{j}}(u, \nabla u) \partial_{j} \overline{\partial^{\alpha} J^{\beta} u} & \\ +\sum_{j=1}^{N} G_{4, j}(u, \nabla u) \partial_{j} \partial^{\alpha} J^{\beta} u+\sum_{j=1}^{N} G_{4, j}^{\prime}(u, \nabla u) \partial_{j} \overline{\partial^{\alpha} J^{\beta} u,} & \text { if } N=2,\end{cases}
$$$$
G_{0}(u, \nabla u)=\int_{0}^{1} \frac{\partial F}{\partial u}(\theta u, \theta \nabla u) d \theta, \quad G_{0}^{\prime}(u, \nabla u)=\int_{0}^{1} \frac{\partial F}{\partial u}(\theta u, \theta \nabla u) d \theta,
$$$$
G_{j}(u, \nabla u)=\int_{0}^{1} \frac{\partial F}{\partial q_{j}}(\theta u, \theta \nabla u) d \theta, \quad G_{j}^{\prime}(u, \nabla u)=\int_{0}^{1} \frac{\partial F}{\partial \bar{q}_{j}}(\theta u, \theta \nabla u) d \theta,
$$$$
G_{4, j}(u, \nabla u)=\int_{0}^{1} \frac{\partial F_{4}}{\partial q_{j}}(\theta u, \theta \nabla u) d \theta, \quad G_{4, j}^{\prime}(u, \nabla u)=\int_{0}^{1} \frac{\partial F_{4}}{\partial \bar{q}_{j}}(\theta u, \theta \nabla u) d \theta
$$

for $j=1, \cdots, N$. Using Lemma 3.3 and the Sobolev embedding $H^{[n / 2]+1}\left(\mathbf{R}^{n}\right) \hookrightarrow$ $L^{\infty}\left(\mathbf{R}^{n}\right)$, we have

$$
\begin{aligned}
&\|u(t)\|_{W^{2, \infty}} \leq C(1+t)^{-3 / 4} X_{7}^{2}(t), \\
&\|J u(t)\|_{W^{1, \infty}} \leq C X_{7}^{2}(t),
\end{aligned}
$$


for $N=2, t \in[0, T]$ and $u \in C\left([0, T] ; H^{7}\right) \cap C\left([0, T] ; H^{3,1}\right)$,

and

$$
\begin{aligned}
& \|u(t)\|_{W^{3, \infty}} \leq C(1+t)^{-11 / 8} X_{m 0}^{N}(t), \\
& \|J u(t)\|_{W^{2, \infty}} \leq C(1+t)^{-1 / 2} X_{m_{0}}^{N}(t), \\
& \left\|J^{2} u(t)\right\|_{W^{1, \infty}} \leq C(1+t)^{1 / 4} X_{m_{0}}^{N}(t),
\end{aligned}
$$

for $N \geq 3, t \in[0, T]$ and $u \in \bigcap_{j=0}^{2} C\left([0, T] ; H^{m 0-2 j, j}\right)$.

We use the abridged notations of the nonlinear terms, i.e., we abbreviate $F(u(t), \nabla u(t))$ as $F(t)$ for instance. The properties of the nonlinear term $F(u, \nabla u)$ are the following.

\section{Lemma 3.4.}

(1) Let $N \geq 3, m \geq m_{0}, p \geq 3$ and $R$ be an arbitrary positive constant. Then there exists a constant $C=C(R)>0$ such that

$$
\begin{array}{r}
\|F(t)\|_{H^{m-1}}+\sum_{|\alpha|=m}\left\|\left(\partial^{\alpha} F-P_{\alpha}\right)(t)\right\|_{L^{2}} \leq C(1+t)^{-11 / 4} X_{m}^{N}(t)^{3}, \\
\|J F(t)\|_{H^{m-3}}+\sum_{\substack{|\alpha|=m-2 \\
|\beta|=1}}\left\|\left(\partial^{\alpha} J^{\beta} F-P_{\alpha \beta}\right)(t)\right\|_{L^{2}} \leq C(1+t)^{-7 / 4} X_{m}^{N}(t)^{3}, \\
\left\|J^{2} F(t)\right\|_{H^{m-5}}+\sum_{\substack{|\alpha|=m-4 \\
|\beta|=2}}\left\|\left(\partial^{\alpha} J^{\beta} F-P_{\alpha \beta}\right)(t)\right\|_{L^{2}} \leq C(1+t)^{-3 / 4} X_{m}^{N}(t)^{3},
\end{array}
$$

for $u \in \bigcap_{j=0}^{2} C\left([0, T] ; H^{m-2 j, j}\right)$ satisfying $\sup _{t \in[0, T]} X_{m}^{N}(t) \leq R$.

(2) Let $N=2, m \geq 7, p \geq 3, F_{3}(u, q)$ satisfy the gauge invariance (1.10) and $R$ be an arbitrary positive constant. Then there exists a constant $C=C(R)>0$ such that

$$
\begin{gathered}
\|F(t)\|_{H^{m-1}}+\sum_{|\alpha|=m}\left\|\left(\partial^{\alpha} F-P_{\alpha}\right)(t)\right\|_{L^{2}} \leq C(1+t)^{-3 / 2} X_{m}^{2}(t)^{3}, \\
\|J F(t)\|_{H^{2}}+\sum_{\substack{|\alpha|=3 \\
|\beta|=1}}\left\|\left(\partial^{\alpha} J^{\beta} F-P_{\alpha \beta}\right)(t)\right\|_{L^{2}} \leq C(1+t)^{-21 / 20} X_{m}^{2}(t)^{3},
\end{gathered}
$$

for $u \in C\left([0, T] ; H^{m}\right) \cap C\left([0, T] ; H^{3,1}\right)$ satisfying $\sup _{t \in[0, T]} X_{m}^{2}(t) \leq R$. 
Proof. Let us consider the part (1). In the same way as in Lemma 3.2 we have

$$
\|F(t)\|_{H^{m-1}}+\sum_{|\alpha|=m}\left\|\left(\partial^{\alpha} F-P_{\alpha}\right)(t)\right\|_{L^{2}} \leq C\|u(t)\|_{W^{1, \alpha}}^{2}\|u(t)\|_{H^{m}}
$$

Then (3.14) yields (3.17).

We have only to see $J F$ as

$$
\begin{aligned}
& J F=Q_{0}(u, \nabla u, J u, \nabla J u)+(1+t) C_{0}\left(u, \nabla u, \nabla^{2} u\right), \\
& \left.Q_{0}=\text { (quadratic term of }(u, \nabla u)\right) \times(u, \nabla u, J u, \nabla J u), \\
& C_{0}=\text { cubic term of }\left(u, \nabla u, \nabla^{2} u\right) .
\end{aligned}
$$

In the same manner as in Lemma 3.2 we have

$$
\begin{aligned}
\left\|Q_{0}(t)\right\|_{H^{m-3}} & \leq C \sum_{n=0}^{m-3}\|u(t)\|_{W^{1, \infty}}^{2-\frac{n}{m-3}}\|u(t)\|_{H^{m-2}}^{\frac{n}{m-3}}\|J u(t)\|_{W^{1, \infty}}^{\frac{n}{m-3}}\|J u(t)\|_{H^{m-2}}^{1-\frac{n}{m-3}} \\
& \leq C(1+t)^{-15 / 8} X_{m}^{N}(t)^{3} \\
\left\|(1+t) C_{0}(t)\right\|_{H^{m-2}} & \leq C(1+t)^{-7 / 4} X_{m}^{N}(t)^{3} .
\end{aligned}
$$

Then we get

$$
\|J F(t)\|_{H^{m-3}} \leq C(1+t)^{-7 / 4} X_{m}^{N}(t)^{3} .
$$

Let $\alpha$ and $\beta \in\left(\mathbf{Z}_{+}\right)^{N}$ satisfy $|\alpha|=m-2$ and $|\beta|=1$ respectively. The simple calculation gives

$$
\partial^{\alpha} J^{\beta} F-P_{\alpha \beta}=\left(\partial^{\alpha} P_{0 \beta}-P_{\alpha \beta}\right)+\sum_{\left|\alpha^{\prime}\right|=m-2}\left(\partial^{\alpha^{\prime}} Q_{0 \alpha^{\prime}}(1+t)+\partial^{\alpha^{\prime}} C_{0 \alpha^{\prime}}\right)
$$

We remark that the structures of $Q_{0 \alpha^{\prime}}$ and $C_{0 \alpha^{\prime}}$ are the same as those of $Q_{0}$ and $C_{0}$ respectively, and furthermore $Q_{0 \alpha^{\prime}}$ does not contain $\nabla J u$. Then we obtain

$$
\sum_{\substack{|\alpha|=m-2 \\|\beta|=1}}\left\|\left(\partial^{\alpha} J^{\beta} F-P_{\alpha \beta}\right)(t)\right\|_{L^{2}} \leq C(1+t)^{-7 / 4} X_{m}^{N}(t)^{3}
$$

(3.22) - (3.23) shows (3.18).

Next we obtain (3.19). We can see $J^{2} F$ as 


$$
\begin{aligned}
J^{2} F= & Q_{1}\left(u, \nabla u, J u, J^{2} u, \nabla J^{2} u\right) \\
& +(1+t) Q_{2}\left(u, \nabla u, \nabla^{2} u, J u, \nabla J u, \nabla^{2} J u\right) \\
& +(1+t)^{2} C_{1}\left(u, \nabla u, \nabla^{2} u, \nabla^{3} u\right), \\
Q_{1}= & \text { (quadratic term of }(u, \nabla u)) \times\left(J u, J^{2} u, \nabla J^{2} u\right), \\
Q_{2}= & \text { (quadratic term of } \left.\left(u, \nabla u, \nabla^{2} u\right)\right) \times\left(u, \nabla u, J u, \nabla J u, \nabla^{2} J u\right), \\
C_{1}= & \text { cubic term of }\left(u, \nabla u, \nabla^{2} u, \nabla^{3} u\right) .
\end{aligned}
$$

Then we have

$$
\begin{aligned}
&\left\|Q_{1}(t)\right\|_{H^{m-5}} \leq C \sum_{n=0}^{m-5}\|u(t)\|_{W^{1, \infty}}^{2-\frac{n}{m^{-5}}}\|u(t)\|_{H^{m-4}}^{\frac{n}{m^{m-5}}} \\
& \times \sum_{k=1,2}^{n}\left\|J^{k} u(t)\right\|_{W^{1, \infty}}^{\frac{n}{m-5}} \sum_{k^{\prime}=1,2}\left\|J^{k^{\prime}} u(t)\right\|_{H^{m-4}}^{1-\frac{n}{m-5}} \\
& \leq C(1+t)^{-9 / 8} X_{m}^{N}(t)^{3} \\
&\left\|(1+t) Q_{2}(t)\right\|_{H^{m-4}} \leq C(1+t)^{-7 / 8} X_{m}^{N}(t)^{3} . \\
&\left\|(1+t)^{2} C_{1}(t)\right\|_{H^{m-4}} \leq C(1+t)^{-3 / 4} X_{m}^{N}(t)^{3}
\end{aligned}
$$

Then we get

$$
\left\|J^{2} F(t)\right\|_{H^{m-5}} \leq C(1+t)^{-3 / 4} X_{m}^{N}(t)^{3}
$$

For any $\alpha$ and $\beta \in\left(\mathbb{Z}_{+}\right)^{N}$ satisfy $|\alpha|=m-2$ and $|\beta|=1$, we have

$$
\begin{aligned}
\partial^{\alpha} J^{\beta} F-P_{\alpha \beta}= & \left(\partial^{\alpha} P_{0 \beta}-P_{\alpha \beta}\right) \\
& +\sum_{\left|\alpha^{\prime}\right|=m-4}\left(\partial^{\alpha^{\prime}} Q_{1 \alpha^{\prime}}+(1+t) \partial^{\alpha^{\prime}} C_{2 \alpha^{\prime}}+(1+t)^{2} \partial^{\alpha^{\prime}} C_{1 \alpha^{\prime}}\right)
\end{aligned}
$$

The structures of $Q_{1 \alpha^{\prime}}, Q_{2 \alpha^{\prime}}$ and $C_{1 \alpha^{\prime}}$ are the same as those of $Q_{1}, Q_{2}$ and $C_{1}$ respectively, and $Q_{1 \alpha^{\prime}}$ does not contain $\nabla J^{2} u$. Then we obtain

$$
\sum_{\substack{|\alpha|=m-4 \\|\beta|=2}}\left\|\left(\partial^{\alpha} J^{\beta} F-P_{\alpha \beta}\right)(t)\right\|_{L^{2}} \leq C(1+t)^{-3 / 4} X_{m}^{N}(t)^{3} .
$$

(3.24) - (3.25) shows (3.19).

Now we consider the part (2). In the same way as (3.17) we can show (3.20) with (3.12) and (3.13). But there are some differences between the proofs of (3.18) and of (3.21). First we remark that because $F_{3}(u, q)$ satisfies (1.4), the loss of decay does not take place in $F_{3}(u, \nabla u)$. In fact we have 


$$
\begin{aligned}
J F_{3}(u, \nabla u)= & \frac{\partial F_{3}}{\partial u}(u, \nabla u) J u-\frac{\partial F_{3}}{\partial \bar{u}}(u, \nabla u) \overline{J u} \\
& +\sum_{j=1}^{N} \frac{\partial F_{3}}{\partial q_{j}}(u, \nabla u) J \partial_{j} u-\sum_{j=1}^{N} \frac{\partial F_{3}}{\partial \bar{q}_{j}}(u, \nabla u) \overline{J \partial_{j} u} .
\end{aligned}
$$

Then the simple calculation gives

$$
\begin{aligned}
& J F=Q_{3}(u, \nabla u, J u, \nabla J u)+(1+t) C_{3}\left(u, \nabla u, \nabla^{2} u\right), \\
& Q_{3}=Q_{4}(u, \nabla u) \times(u, \cdot \nabla u, J u, \nabla J u), \\
& Q_{4}=\text { quadratic term of }(u, \nabla u), \\
& C_{3}=\text { the fourth order term of }\left(u, \nabla u, \nabla^{2} u\right) .
\end{aligned}
$$

In the same way as the evaluation to $C_{0}(t)$ we can estimate $C_{3}(t)$. On the other hand, if we try to get the bound of $Q_{3}(t)$ by the same technique used for $Q_{0}(t)$, then the lack of decay occurs. To avoid this difficulty we make use of another estimates. We have

$$
\left\|Q_{3}(t)\right\|_{H^{2}} \leq C\left\|Q_{4}(t)\right\|_{W^{2, \infty}}\left(\|u(t)\|_{H^{2}}+\|J u(t)\|_{H^{3}}\right)
$$

Similarly when we try to obtain

$$
\sum_{\substack{\alpha|=3\\| \boldsymbol{\beta} \mid=1}}\left\|\left(\partial^{\alpha} J^{\beta} F-P_{\alpha \beta}\right)(t)\right\|_{L^{2}} \leq C(1+t)^{-21 / 20} X_{m}^{2}(t)^{3},
$$

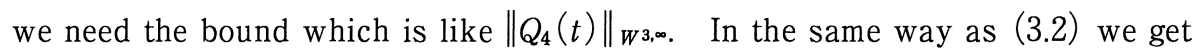

$$
\left\|Q_{4}(t)\right\|_{W^{3, \infty}} \leq C \sum_{n=0}^{3}\|u(t)\|_{H^{7}}^{\frac{n}{5}}\|u(t)\|_{W^{1, \infty}}^{2-\frac{n}{5}} \leq C(1+t)^{-21 / 20} X_{m}^{2}(t)^{2}
$$

Substituting (3.28) into (3.26) we obtain

$$
\left\|Q_{3}(t)\right\|_{H^{2}} \leq C(1+t)^{-21 / 20} X_{m}^{2}(t)^{3} .
$$

Similarly we can obtain (3.27). (3.27) - (3.29) shows (3.21). Proof of Lemma 3.4 is finished.

Remark 3.1. Let $N=2$. In general, if $m>3 l^{\prime} / 2+2, l^{\prime} \in \mathbf{N}$, then we can obtain 


$$
\begin{aligned}
\|J F(t)\|_{H^{\prime}-1} & +\sum_{\substack{|\alpha|=l^{\prime} \\
|\beta|=1\\
}}\left\|\left(\partial^{\alpha} J^{\beta} F-P_{\alpha \beta}\right)(t)\right\|_{L^{2}} \\
& \leq C(1+t)^{-(1+4 \varepsilon 1)}\left(\left\|u(t)_{H^{m}}+\right\| J u(t) \|_{H^{\prime}}\right)^{3}
\end{aligned}
$$

for $u \in C\left([0, T] ; H^{m}\right) \cap C\left([0, T] ; H^{l^{\prime}, 1}\right)$ satisfying

$$
\sup _{t \in[0, T]}\left(\|u(t)\|_{H^{m}}+\|J u(t)\|_{H^{r^{\prime}}}\right) \leq R \text { with some } R>0
$$

where $\varepsilon_{1}=\frac{m-3 l^{\prime} / 2-2}{8(m-2)}>0$.

\section{\$4. Proof of Theorem 1.1}

In this section we prove Theorem 1.1 by the parabolic regularization and the uniform estimates.

At the first step we consider

$$
\begin{array}{cl}
\partial_{t} u^{\varepsilon}-(\varepsilon+i) \Delta u^{\varepsilon}=F\left(u^{\varepsilon}, \nabla u^{\varepsilon}\right) & \text { in } \quad(0, \infty) \times \mathbb{R}^{N}, \\
u^{\varepsilon}(0, x)=u_{0}(x) & \text { in } \mathbb{R}^{N},
\end{array}
$$

where $\varepsilon \in(0,1]$. Because the parabolic regularization resolve the loss of derivatives, we can easily solve (4.1)-(4.2)

Lemma 4.1. Let $m$ be an integer $>N / 2+1$. Then for any $u_{0} \in H^{m}$, there exists a time $T_{\varepsilon}=T\left(\varepsilon,\left\|u_{0}\right\|_{H^{[N / 2]+2}}\right.$ suth that the initial value problem (4.1) - (4.2) possesses a unique solution $u^{\varepsilon} \in C\left(\left[0, T_{\varepsilon}\right) ; H^{m}\right)$. Moreover the map $u_{0} \in H^{\mathrm{m}} \mapsto u^{\varepsilon}$ $\in C\left(\left[0, T_{\varepsilon}\right) ; H^{m}\right)$ is continuous.

Proof. It is easy to verify Lemma 4.1 with (3.2) and (3.4). See e.g., [1] or $[2]$.

To get a local solution to (1.1) - (1.2), we obtain the uniform estimates on $\left\{u^{\varepsilon}\right\}_{\varepsilon \in(0,1]}$ by the energy inequality in Lemma 2.4. Let $l \in \mathbb{N}$ be the same integer as in $\S 2$. We put $m_{1}=[N / 2]+l+4$ and let $m$ be an integer $\geq m_{1}$. As we mentioned in Remark 2.1, if $\left\|u^{\varepsilon}(t)\right\|_{W^{1, \infty}} \leq R<+\infty$, then there exists a constant $M=M(R, F)>0$ such that 


$$
\begin{aligned}
\left|\operatorname{Im} \frac{\partial F}{\partial q_{j}}\left(u^{\varepsilon}(t), \nabla u^{\varepsilon}(t)\right)\right| & \leq C_{R} \sup _{\check{x}, \in \mathbf{R}^{N-1}}\left(\left|u^{\varepsilon}(t, x)\right|+\left|\nabla u^{\varepsilon}(t, x)\right|\right)^{2} \leq \phi_{j}^{\varepsilon}\left(t, x_{j}\right), \\
\phi_{j}^{\varepsilon}\left(t, x_{j}\right) & =M \sum_{|\alpha| \leqq\left[\frac{N-1}{2}\right]+2} \int_{\mathbf{R}^{N-1}}\left|\partial^{\alpha} u^{\varepsilon}(t, x)\right|^{2} d \check{x}_{j} .
\end{aligned}
$$

We define

$$
\begin{aligned}
& v_{\alpha}^{\varepsilon}={ }^{t}\left(\partial^{\alpha} u^{\varepsilon}, \partial^{\alpha} \overline{u^{\varepsilon}}\right), \quad v_{\alpha, 0}={ }^{t}\left(\partial^{\alpha} u_{0}, \partial^{\alpha} \overline{u_{0}}\right), \quad \text { for }|\alpha|=m \text {, } \\
& b_{11,, \varepsilon}(t, x)=-\frac{\partial F}{\partial q_{j}}\left(u^{\varepsilon}, \nabla u^{\varepsilon}\right), \quad b_{12 j, \varepsilon}(t, x)=-\frac{\partial F}{\partial \bar{q}_{j}}\left(u^{\varepsilon}, \nabla u^{\varepsilon}\right), \\
& b_{21 j, \varepsilon}(t, x)=\overline{\frac{\partial F}{\partial \bar{q}_{j}}\left(u^{\varepsilon}, \nabla u^{\varepsilon}\right)}, \quad b_{22 j, \varepsilon}(t, x)=\overline{\frac{\partial F}{\partial q_{j}}\left(u^{\varepsilon}, \nabla u^{\varepsilon}\right)}, \\
& b_{\varepsilon}(t, x, \xi)=\sum_{j=1}^{N}\left[\begin{array}{ll}
b_{11, \varepsilon}(t, x) & b_{12 j, \varepsilon}(t, x) \\
b_{21 j, \varepsilon}(t, x) & b_{22 j, \varepsilon}(t, x)
\end{array}\right] \xi_{\jmath} \\
& b_{\varepsilon}^{\text {diag }}(t, x, \xi)=\sum_{j=1}^{N}\left[\begin{array}{cc}
b_{11 j, \varepsilon}(t, x) & 0 \\
0 & b_{22 j, \varepsilon}(t, x)
\end{array}\right] \xi_{j} \text {, } \\
& K_{\varepsilon}(t)=k_{\varepsilon}(t, x, D), \quad k_{\varepsilon}(t, x, \xi)=\left[\begin{array}{cc}
k_{1, \varepsilon}(t, x, \xi) & 0 \\
0 & k_{1, \varepsilon}(t, x, \xi)^{-1}
\end{array}\right], \\
& k_{1, \varepsilon}(t, x, \xi)=\exp \left(-\sum_{j=1}^{N} \int_{0}^{x,} \phi_{j}^{\varepsilon}(t, s) d s \xi_{j}\left\langle\xi_{j}\right\rangle^{-1}\right) \text {, } \\
& \Lambda_{\varepsilon}(t)=I+\tilde{\Lambda}_{\varepsilon}(t), \quad \tilde{\Lambda}_{\varepsilon}(t)=\tilde{\lambda}_{\varepsilon}(t, x, D), \\
& \tilde{\lambda}_{\varepsilon}(t, x, \xi)=\frac{1}{2} \sum_{j=1}^{N}\left[\begin{array}{cc}
0 & b_{12 j, \varepsilon}(t, x) \\
-b_{21 j, \varepsilon}(t, x) & 0
\end{array}\right] \xi_{j}\langle\xi\rangle-2, \\
& N_{m}^{\varepsilon}(t)=\sum_{|\alpha|=m}\left\|K_{\varepsilon}(t) \Lambda_{\varepsilon}(t) v_{\alpha}^{\varepsilon}(t)\right\|+\left\|u^{\varepsilon}(t)\right\|_{H^{m-1}} \\
& \left.\delta_{m}=N_{m}^{\varepsilon}(0)=\sum_{|\alpha|=m}\left\|K_{\varepsilon}(0) \Lambda_{\varepsilon}(0) v_{\alpha, 0}\right\|+\left\|u_{0}\right\|_{H^{m-1}} \quad \text { (ind. of } \varepsilon \in(0,1]\right) \\
& f_{\alpha}^{\varepsilon}={ }^{t}\left(f_{\alpha, 1}^{\varepsilon}, f_{\alpha, 2}^{\varepsilon}\right), \quad f_{\alpha, 1}^{\varepsilon}=\overline{f_{\alpha, 2}^{\varepsilon}} \quad \text { for } \quad|\alpha|=m, \\
& f_{\alpha, 1}^{\varepsilon}=\partial^{\alpha} F\left(u^{\varepsilon}, \nabla u^{\varepsilon}\right)-\sum_{j=1}^{N} \frac{\partial F}{\partial q_{j}}\left(u^{\varepsilon}, \nabla u^{\varepsilon}\right) \partial_{j} \partial^{\alpha} u^{\varepsilon}-\sum_{j=1}^{N} \frac{\partial F}{\partial \bar{q}_{j}}\left(u^{\varepsilon}, \nabla u^{\varepsilon}\right) \partial_{j} \partial^{\alpha} \bar{u}^{\varepsilon}
\end{aligned}
$$


In the same way as in $\S 2$, we define $B_{b_{\varepsilon}}(t), B_{K_{\varepsilon}}(t), B_{\phi^{\varepsilon}}^{0}(t), B_{\phi^{\varepsilon}}^{1}(t)$ and $B_{\phi^{\varepsilon}}^{\infty}(t)$. It is easy to check that there exists a non-decreasing function $A(\cdot)$ on $[0, \infty)$ such that

$$
B_{b_{\varepsilon}}(t), B_{K_{\varepsilon}}(t), B_{\phi^{\varepsilon}}^{o}(t), B_{\phi^{\varepsilon}}^{1}(t), B_{\phi^{\varepsilon}}^{\infty}(t) \leq A\left(\left\|u^{\varepsilon}(t)\right\|_{H^{m_{1}-1}}\right)
$$

(3.2) - (3.3) implies

$$
\left\|F\left(u^{\varepsilon}(t), \nabla u^{\varepsilon}(t)\right)\right\|_{H^{m-1}}+\sum_{|\alpha|=m}\left\|f_{\alpha}^{\varepsilon}(t)\right\| \leq A\left(\left\|u^{\varepsilon}(t)\right\|_{H^{m_{1}-1}}\right)\left\|u^{\varepsilon}(t)\right\|_{H^{m}} .
$$

Let $T_{\varepsilon}^{*}$ be a time defined by

$$
T_{\varepsilon}^{*}=\sup \left\{T>0 \mid N_{m_{1}}^{\varepsilon}(t)<2 \delta_{m_{1}}, \quad 0 \leq t<T\right\}
$$

Lemma 4.1 ensures $T_{\varepsilon} *>0$. In view of the Sobolev embedding, there exists a constant $R=R\left(\delta_{m_{1}}\right)>0$ such that $\left\|u^{\varepsilon}(t)\right\|_{W_{1, \infty}} \leq R$ for $t \in\left[0, T_{\varepsilon}^{*}\right]$ and $\varepsilon \in(0,1]$. Moreover there exists a constant $C\left(\delta_{m_{1}}\right)>0$ such that

$$
\begin{gathered}
B_{b_{\varepsilon}}(t), B_{K_{\varepsilon}}(t), B_{\phi^{\varepsilon}}^{0}(t), B_{\phi^{\varepsilon}}^{1}(t), B_{\phi^{\varepsilon}}^{\infty}(t) \leq C\left(\delta_{m_{1}}\right), \\
C\left(\delta_{m_{1}}\right)^{-1} N_{m}^{\varepsilon}(t) \leq\left\|u^{\varepsilon}(t)\right\|_{H^{m}} \leq C\left(\delta_{m_{1}}\right) N_{m}^{\varepsilon}(t), \\
\left\|F\left(u^{\varepsilon}(t), \nabla u^{\varepsilon}(t)\right)\right\|_{H^{m-1}}+\sum_{|\alpha|=m}\left\|K_{\varepsilon}(t) \Lambda_{\varepsilon}(t) f_{\alpha}^{\varepsilon}(t)\right\| \leq C\left(\delta_{m_{1}}\right) N_{m}^{\varepsilon}(t)
\end{gathered}
$$

for $t \in\left[0, T_{\varepsilon}^{*}\right]$ and $\varepsilon \in(0,1] . \quad v_{\alpha}^{\varepsilon}$ satisfies

$$
\begin{gathered}
\left(I \partial_{t}+\varepsilon I|D|^{2}+i\left(a(D)+b_{\varepsilon}(t, x, D)\right)\right) v_{\alpha}^{\varepsilon}=f_{\alpha}^{\varepsilon} \\
v_{\alpha}^{\varepsilon}(0, x)=v_{\alpha, 0}(x) .
\end{gathered}
$$

The local wellposedness for (4.1) - (4.2) ensures the validity of the energy estimates. Here we note that

$$
\begin{gathered}
\varepsilon \Lambda_{\varepsilon}(t) I|D|^{2}=\varepsilon I|D|^{2} \Lambda_{\varepsilon}(t)+\varepsilon R_{2, \varepsilon}(t), \\
R_{2, \varepsilon}(t)=\tilde{\Lambda}_{\varepsilon}(t)|D|^{2}-|D|^{2} \tilde{\Lambda}_{\varepsilon}(t), \quad\left\|R_{2, \varepsilon}(t)\right\|_{\mathscr{L}\left(\left(L^{2) 2}\right)\right.} \leq C\left(\delta_{m 1}\right) \text { for } t \in\left[0, T_{\varepsilon}^{*}\right] . \\
(2.6)-(2.7) \text { implies }
\end{gathered}
$$




$$
\begin{gathered}
\left(I \partial_{t}+\varepsilon I|D|^{2}+i a(D)+q_{\varepsilon}(t, x, D)\right) K_{\varepsilon}(t) \Lambda_{\varepsilon}(t) v_{\alpha}^{\varepsilon}+R_{3, \varepsilon}(t) v_{\alpha}^{\varepsilon}=K_{\varepsilon}(t) \Lambda_{\varepsilon}(t) f_{\alpha}^{\varepsilon}, \\
q_{\varepsilon}(t, x, D)=2(I+i \varepsilon \tilde{I}) \sum_{j=1}^{N} \phi_{j}^{\varepsilon}\left(t, x_{j}\right) D_{j}^{2}\left\langle D_{j}\right\rangle^{-1}+b_{\varepsilon}^{\text {diag }}(t, x, D) \\
R_{3, \varepsilon}=R_{1, \varepsilon}(t)+\varepsilon K_{\varepsilon}(t) R_{2, \varepsilon}(t), \quad\left\|R_{3, \varepsilon}(t)\right\|_{\varphi\left(\left(L^{2}\right) 2\right)} \leq C\left(\delta_{m_{1}}\right) \text { for } t \in\left[0, T_{\varepsilon}^{*}\right]
\end{gathered}
$$

where $R_{1, \varepsilon}(t)$ corresponds to $R_{1}(t)$ (see $\S 2$ ) and $\widetilde{I}$ is a $2 \times 2$ diagonal matrix of $(1,-1)$. Noting $(4.3)$, in the same manner as $(2.10)$ we get

$$
\frac{d}{d t}\left\|K_{\varepsilon}(t) \Lambda_{\varepsilon}(t) v_{\alpha}^{\varepsilon}(t)\right\| \leq C\left(\delta_{m_{1}}\right) N_{m}^{\varepsilon}(t)+\left\|K_{\varepsilon}(t) \Lambda_{\varepsilon}(t) f_{\alpha}^{\varepsilon}(t)\right\|
$$

On the other hand we have

$$
\frac{d}{d t}\left\|u^{\varepsilon}(t)\right\|_{H^{m-1}} \leq\left\|F\left(u^{\varepsilon}(t), \nabla u^{\varepsilon}(t)\right)\right\|_{H^{m-1}}
$$

Combining (4.4), (4.5) and (4.6) we obtain

$$
\frac{d}{d t} N_{m}^{\varepsilon}(t) \leq C\left(\delta_{m_{1}}\right) N_{m}^{\varepsilon}(t), \quad \text { for } t \in\left[0, T_{\varepsilon}^{*}\right]
$$

The Gronwall inequality yields

$$
N_{m}^{\varepsilon}(t) \leq \delta_{m} \exp \left(C\left(\delta_{m_{1}}\right) t\right) \text { for } t \in\left[0, T_{\varepsilon}^{*}\right]
$$

If we put $m=m_{1}$ and $t=T_{\varepsilon}{ }^{*}$ in (4.7), then we have

$$
T_{\varepsilon}^{*} \geq T \equiv(\log 2) C\left(\delta_{m_{1}}\right)^{-1}>0
$$

Hence $\left\{u^{\varepsilon}\right\}_{\varepsilon \in(0,1]}$ is bounded in $L^{\infty}\left(0, T ; H^{m}\right)$. Then the standard compactness argument implies that there exists a solution $u \in L^{\infty}\left(0, T ; H^{m}\right)$ to $(1.1)-(1.2)$ (see [1] for instance). The uniqueness and the continuity in the time variable are proved by the same energy method. This completes the proof of Theorem 1.1 .

\section{\$5. Proof of Theorem 1.2}

In this section we prove Theorem 1.2. In view of Theorem 1.1, we have only to obtain the a priori estimates on $\|u(t)\|_{H^{m_{1}}}$. Let $l$ be the same integer as in $\S 2$. We take $m_{2}$ as $m_{2}=[(N+1) / 2]+l+5 \geq m_{1}+1$. Then it is sufficent to get the $a$ 
priori estimates on $\|u(t)\|_{H^{m_{2}}}$. Since we assume $u_{0} \in H^{m_{2}+2}$, Theorem 1.1 and Lemma 2.4 ensure the validity of the energy estimates in $H^{m_{2}}$.

Let $u$ be a solution to (1.1) - (1.2) satisfying

$$
u \in \bigcap_{j=0}^{2} C\left([0, T] ; H^{m-2 j, j}\right), \sup _{t \in[0, T]}\|u(t)\|_{W^{1, \infty}} \leq R_{0}
$$

with some $T, R_{0}>0$ and some $m \in \mathbb{N} \geq m_{2}+2$. The local existence in this weighted Sobolev space is proved by the same method proving Theorem 1.1 or by [2]. To carry out the energy estimates, we introduce some notations

$$
\begin{aligned}
& b_{11 j}^{1}(t, x)=-\frac{\partial F}{\partial q_{j}}(u, \nabla u), \quad b_{12 j}^{1}(t, x)=-\frac{\partial F}{\partial \bar{q}_{j}}(u, \nabla u), \\
& b_{21 j}^{1}(t, x)=\overline{\frac{\partial F}{\partial \bar{q}_{j}}(u, \nabla u)}, \quad b_{22 j}^{1}(t, x)=\overline{\frac{\partial F}{\partial q_{j}}(u, \nabla u)}, \\
& b_{11 j}^{2}(t, x)=-G_{j}(u, \nabla u), \quad b_{12 j}^{2}(t, x)=-G_{j}^{\prime}(u, \nabla u), \\
& b_{21 j}^{2}(t, x)=\overline{G_{j}^{\prime}(u, \nabla u)}, \quad b_{22 j}^{2}(t, x)=\overline{G_{j}(u, \nabla u)}, \quad j=1, \cdots, N, \\
& b^{n}(t, x, \xi)=\sum_{j=1}^{N}\left[\begin{array}{ll}
b_{11 j}^{n}(t, x) & b_{12 j}^{n}(t, x) \\
b_{21 j}^{n}(t, x) & b_{22 j}^{n}(t, x)
\end{array}\right] \xi_{j}, \quad n=1,2
\end{aligned}
$$

In the same way as in $\S 2$, we define $B_{b^{n}}(t), n=1,2$ and we put $B_{b}(t)=B_{b^{1}}(t)+$ $B_{b^{2}}(t)$. By the Gagliardo-Nirenberg inequality (the interpolation between $W^{1, \infty}$ and $H^{m_{2}-1}$ for $W^{l+3, \infty}$ ) and (3.14), we have

$$
\begin{gathered}
B_{b}(t) \leq C_{R_{0}}(1+t)^{-(1+\varepsilon)} X_{m_{2}-1}^{N}(t)^{2} \text { for } t \in[0, T], \\
\varepsilon=\frac{7}{4} \frac{m_{2}-N / 2-2 l / 7-4}{m_{2}-N / 2-4}>0 .
\end{gathered}
$$

Using (3.6), in the same way as (4.3), we have

$$
\begin{array}{r}
\left|\operatorname{Im} b_{h h j}^{n}(t, x)\right| \leq \phi_{j}\left(t, x_{j}\right), \text { for }(t, x) \in[0, T] \times \mathbb{R}^{N}, n, h=1,2, j=1, \cdots, N, \\
\phi_{j}\left(t, x_{j}\right)=M(1+t)^{-3 / 2} \sum_{\substack{|\alpha| \leq\left[\frac{N+1}{2}\right]+1 \\
|\beta| \leq 1}} \int_{\mathbb{R}^{N-1}}\left|\partial^{\alpha} J^{\beta} u(t, x)\right|^{2} d \check{x}_{j}, j=1, \cdots, N,
\end{array}
$$


where $M=M\left(R_{0}, F\right)>0$ is a sufficiently large constant. We introduce the pseudodifferential operators defined as follows.

$$
\begin{aligned}
& K(t)=k(t, x, D), \quad k(t, x, \xi)=\left[\begin{array}{cc}
k_{1}(t, x, \xi) & 0 \\
0 & k_{1}(t, x, \xi)-1
\end{array}\right] \\
& k_{1}(t, x, \xi)=\exp \left(-\sum_{j=1}^{N} \int_{0}^{x} \phi_{j}(t, s) d s \xi_{j}\left\langle\xi_{j}\right\rangle^{-1}\right) \text {, } \\
& \Lambda_{n}(t)=I+\tilde{\Lambda}_{n}(t), \quad \tilde{\Lambda}_{n}(t)=\tilde{\lambda}_{n}(t, x, D), \\
& \tilde{\lambda}_{n}(t, x, \xi)=\frac{1}{2} \sum_{j=1}^{N}\left[\begin{array}{cc}
0 & b_{12 j}^{n}(t, x) \\
-b_{21 j}^{n}(t, x) & 0
\end{array}\right] \xi_{j}\langle\xi\rangle^{-2}, \quad n=1,2 \text {. }
\end{aligned}
$$

We also define $B_{K}(t), B_{\phi}^{0}(t), B_{\phi}^{1}(t)$ and $B_{\phi}^{\infty}(t)$. The simple calculation yields

$$
\begin{aligned}
& B_{K}(t), B_{\phi}^{0}(t) \leq A\left(X_{m_{2}-1}^{N}(t)\right), \\
& B_{\phi}^{1}(t), B_{\phi}^{\infty}(t) \leq C_{R_{0}}(1+t)^{-3 / 2} X_{m_{2}-1}^{N}(t)^{2},
\end{aligned}
$$

where $A(\cdot)$ is a non-decreasing function on $[0, \infty)$ depending on $R_{0}$. We put

$$
\begin{array}{cc}
v_{\alpha}={ }^{t}\left(\partial^{\alpha} u, \partial^{\alpha} \bar{u}\right), & v_{\alpha, 0}={ }^{t}\left(\partial^{\alpha} u_{0}, \partial^{\alpha} \overline{u_{0}}\right), \\
f_{\alpha}={ }^{t}\left(f_{\alpha, 1}, f_{\alpha, 2}\right), & f_{\alpha, 1}=\overline{f_{\alpha, 2}} \\
f_{\alpha, 1}=\partial^{\alpha} F-P_{\alpha}, & \text { for }|\alpha|=m_{2},
\end{array}
$$

and

$$
\begin{array}{rlrl}
v_{\alpha \beta} & ={ }^{t}\left(\partial^{\alpha} J^{\beta} u, \overline{\partial^{\alpha} J^{\beta} u}\right), & v_{\alpha \beta, 0}=v_{\alpha \beta}(0)={ }^{t}\left(\partial^{\alpha}\left(x^{\beta} u_{0}\right), \overline{\partial^{\alpha}\left(x^{\beta} u_{0}\right)}\right), \\
f_{\alpha \beta}={ }^{t}\left(f_{\alpha \beta, 1}, f_{\alpha \beta, 2}\right), & f_{\alpha \beta, 1}=\overline{f_{\alpha \beta, 2}} \\
f_{\alpha \beta, 1}=\partial^{\alpha} J^{\beta} F-P_{\alpha \beta}, & \text { for }|\alpha+2 \beta|=m_{2},|\beta|=1,2 .
\end{array}
$$

We evaluate $Y_{m_{2}}^{N}(t)$ which is defined by

$$
\begin{aligned}
Y_{m_{2}}^{N}(t)= & X_{m_{2}-1}^{N}(t)+\sum_{|\alpha|=m_{2}}\left\|K(t) \Lambda_{1}(t) v_{\alpha}(t)\right\| \\
& +\sum_{\substack{|\alpha|=m_{2}-2 \\
|\beta|=1}}\left\|K(t) \Lambda_{2}(t) v_{\alpha \beta}(t)\right\|+(1+t)^{-1 / 4} \sum_{\substack{|\alpha|=m_{2}-4 \\
|\beta|=2}}\left\|K(t) \Lambda_{2}(t) v_{\alpha \beta}(t)\right\| .
\end{aligned}
$$


We suppose that there exists a constant $R>0$ such that

$$
\sup _{t \in[0, T]} Y_{m_{2}}^{N}(t) \leq R
$$

In view of (2.4), (2.5), (5.1), (5.3) and (5.4), we have

$$
C_{R}^{-1} Y_{m_{2}}^{N}(t) \leq X_{m_{2}}^{N}(t) \leq C_{R} Y_{m_{2}}^{N}(t), \quad Y_{m_{2}}^{N}(0) \leq C_{R} \delta
$$

with some constant $C_{R}>0$. Using (3.17), (3.18) and (3.19) and (5.5) we get

$$
\begin{aligned}
& \|F(t)\|_{H^{m_{2}-1}}+\sum_{|\alpha|=m_{2}}\left\|K(t) \Lambda_{1}(t) f_{\alpha}(t)\right\| \leq C_{R}(1+t)^{-11 / 4} R^{3},
\end{aligned}
$$

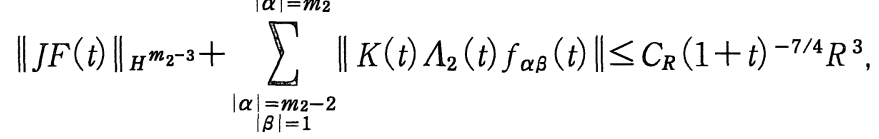

$$
\begin{aligned}
& \left\|J^{2} F(t)\right\|_{H^{m_{2}-5}}+\sum_{\substack{|\alpha|=m_{2}-4 \\
\boldsymbol{\beta} \mid=2}}\left\|K(t) \Lambda_{2}(t) f_{\alpha \beta}(t)\right\| \leq C_{R}(1+t)^{-3 / 4} R^{3} .
\end{aligned}
$$

With (5.1), (5.3) and (5.4) we have

$$
\left(B_{b}(t)+B_{\phi}^{1}(t)+B_{\phi}^{\infty}(t)\right) B_{\text {etc }}(t) \leq C_{R}(1+t)^{-(1+\varepsilon)} R^{2} .
$$

$v_{\alpha}$ and $v_{\alpha \beta}$ satisfy

$$
\begin{gathered}
\left(I \partial_{t}+i\left(a(D)+b^{1}(t, x, D)\right)\right) v_{\alpha}=f_{\alpha}, \\
\left(I \partial_{t}+i\left(a(D)+b^{2}(t, x, D)\right)\right) v_{\alpha \beta}=f_{\alpha \beta},
\end{gathered}
$$

respectively. Using (2.10), (5.2), (5.6), (5.7), (5.8) and (5.9), we obtain

$$
\begin{aligned}
& \frac{d}{d t} \sum_{\substack{|\alpha|=m_{2}\\
}}\left\|K(t) \Lambda_{1}(t) v_{\alpha}(t)\right\| \leq C_{R}(1+t)^{-(1+\varepsilon)} R^{3}, \\
& \frac{d}{d t} \sum_{\substack{|\alpha|=m_{2}-2 \\
|\beta|=1}}\left\|K(t) \Lambda_{2}(t) v_{\alpha \beta}(t)\right\| \leq C_{R}(1+t)^{-(1+\varepsilon)} R^{3}, \\
& \frac{d}{d t} \sum_{\substack{|\alpha|=m_{2}-4 \\
|\beta|=2}}\left\|K(t) \Lambda_{2}(t) v_{\alpha \beta}(t)\right\| \leq C_{R}(1+t)^{-3 / 4} R^{3} .
\end{aligned}
$$

The integration on $[0, t]$ implies 


$$
\begin{aligned}
\sum_{|\alpha|=m_{2}}\left\|K(t) \Lambda_{1}(t) v_{\alpha}(t)\right\|+\sum_{\substack{|\alpha|=m_{2}-2 \\
|\beta|=1}}\left\|K(t) \Lambda_{2}(t) v_{\alpha \beta}(t)\right\| \\
+(1+t)^{-1 / 4} \sum_{\substack{\left|\alpha=m_{2}-4\\
\right| \beta \mid=2}}\left\|K(t) \Lambda_{2}(t) v_{\alpha \beta}(t)\right\| \leq C_{R}\left(\delta+R^{3}\right) .
\end{aligned}
$$

Noting $\left[J, \partial_{t}-i \Delta\right]=0$, we have

$$
\left(\partial_{t}-i \Delta\right) \partial^{\alpha} J^{\beta} u=\partial^{\alpha} J^{\beta} F, \quad \text { with } \quad|\alpha+2 \beta| \leq m_{2}-1, \quad|\beta| \leq 2 .
$$

Making use of (5.6), (5.7) and (5.8) we obtain

$$
X_{m_{2}-1}^{N}(t) \leq C_{R}\left(\delta+R^{3}\right)
$$

Combining (5.10) and (5.11) we get

$$
\sup _{t \in[0, T]} Y_{m_{2}}^{N}(t) \leq C_{R}\left(\delta+R^{3}\right)
$$

Then there exist constants $R_{1}>0$ and $C_{R_{1}}>0$ which are independent of $T>0$, such that

$$
\sup _{t \in[0, T]} Y_{m_{2}}^{N}(t) \leq C_{R_{1}}\left(\delta+R^{3}\right), \quad \text { if } \quad R \leq R_{1}
$$

If we choose $R_{1}$ as $C_{R_{1}} R_{1}^{3} \leq R_{1} / 4$ at the beginning, then we have

$$
\sup _{t \in[0, T]} Y_{m_{2}}^{N}(t) \leq R / 2 \quad \text { if } \quad R \leq R_{1}
$$

provided that $\delta$ is sufficiently small. This completes the proof of Theorem 1.2.

\section{\$6. Proof of Theorem $\mathbf{1 . 3}$}

Finally we prove Theorem 1.3. The outline of its proof is basically the same as that of Theorem 1.2. Let $l$ be the same integer as in $\S 2$. We take $m_{3}$ as $m_{3}=[(3 l+1) / 2]+7 \geq m_{1}+1$. We obtain the a priori estimates in $H^{m_{3}} \cap$ $H^{l+3,1}$. Let $m$ be an integer $\geq m_{3}+2$ and let $u$ be a solution to (1.1) $-(1.2)$ satisfying 


$$
u \in \bigcap_{j=0}^{2} C\left([0, T] ; H^{m-2 j, j}\right)
$$

We define $\phi_{j}\left(t, x_{j}\right)$ by

$$
\phi_{j}\left(t, x_{j}\right)=M(1+t)^{-1} \sum_{\substack{|\alpha| \leqq 1 \\|\beta| \leqq 1}} \int_{\mathbb{R}}\left|\partial^{\alpha} J^{\beta} u(t, x)\right|^{2} d \check{x}_{j}, \quad j=1,2,
$$

with some large constant $M>0$. We introduce $K(t), \Lambda_{n}(t), b^{n}(t, x, \xi), n=1,2$, $B_{K}(t), B_{b}(t), B_{\phi}^{0}(t), B_{\phi}^{1}(t)$ and $B_{\phi}^{\infty}(t)$ in the same manner as in $\S 5$, We put

$$
\begin{aligned}
Z(t) & =Z_{1}(t)+(1+t)^{-\varepsilon} Z_{2}(t), \\
Z_{1}(t) & =\|u(t)\|_{H^{m_{3}-1}}+\|J u(t)\|_{H^{l+2}} \\
Z_{2}(t) & =\sum_{|\alpha|=m_{3}}\left\|K(t) \Lambda_{1}(t) v_{\alpha}(t)\right\|+\sum_{\substack{|\alpha|=l+3 \\
|\beta|=1}}\left\|K(t) \Lambda_{2}(t) v_{\alpha \beta}(t)\right\|, \\
\varepsilon & =\frac{m_{3}-(3 l+1) / 2-6}{8\left(m_{3}-2\right)}>0 .
\end{aligned}
$$

We suppose that there exists a constant $R>0$ such that

$$
\sup _{t \in[0, T]} Z(t) \leq R
$$

Then we have

$$
\begin{gathered}
\left|\operatorname{Im} b_{h h j}^{n}(t, x)\right| \leq \phi_{j}\left(t, x_{j}\right), \quad \text { for }(t, x) \in[0, T] \times \mathbb{R}^{N}, \quad n, h, j=1,2, \\
B_{K}(t), B_{\phi}^{0}(t) \leq C_{R}, B_{\phi}^{1}(t), B_{\phi}^{\infty}(t) \leq C_{R}(1+t)^{-1} R^{2}, B_{b}(t) \leq C_{R}(1+t)^{-(1+2 \varepsilon)} R^{2}, \\
\quad\left(B_{b}(t)+B_{\phi}^{1}(t)+B_{\phi}^{\infty}(t)\right) B_{\text {etc }}(t) \leq C_{R}(1+t)^{-1} R^{2}, \\
\|F(t)\|_{H^{m_{3}-1}}+\sum_{|\alpha|=m_{3}}\left\|K(t) \Lambda_{1}(t) f_{\alpha}(t)\right\| \\
+\|J F(t)\|_{H^{l+2}}+\sum_{\substack{|\alpha|=l+3 \\
|\beta|=1}}\left\|K(t) \Lambda_{2}(t) f_{\alpha \beta}(t)\right\| \leq C_{R}(1+t)^{-(1+\varepsilon)} R^{3} .
\end{gathered}
$$

Here we used (3.30) to get the bound of $B_{b}(t)$. Using (2.10) and $(6.1) \sim(6.4)$, we obtain

$$
\sup _{t \in[0, T]} Z(t) \leq C_{R}\left(\delta+R^{3}\right)
$$

In the same way as the proof of Theorem 1.2, we can obtain the a priori estimates. This completes the proof of Theorem 1.3. 


\section{Acknowledgments}

The author would like to express his sincere gratitude to Professors Y. Ohya and S. Tarama for their constant encouragement and invaluable advices. Especially Professor S. Tarama suggested the author to choose $\phi_{j}\left(t, x_{j}\right)$ as (2.13).

\section{REFERENCES}

[1] Chihara, H., Local existence for the semilinear Schrodinger equations in one space dimension, J. Math Kyoto Univ., 34(1994), 353-367.

[2] - Local existence for semilinear Schrödinger equations, Math. Japon., 42 (1995), 35-52.

[3] Global existence of small solutions to seminear Schrödinger equations with gauge invariance, Publ. RIMS, 31 (1995), 731-753.

[4] Global existence of small solutions to semilinear Schrodinger equations, Comm. P. D. E., 21 (1996), 63-78.

[5] Doi, S., On the Cauchy problem for Schrödinger type equations and the regularity of the solutions, J. Math. Kyoto Univ., 34(1994), 319-328.

[6] Hayashi, N., Global existence of small solutions to quadratic nonlinear Schrödinger equations, Comm. P. D. E., 18(1993), 1109-1124.

[7] Hayashi, N. and Ozawa, T., Remarks on nonlinear Schrödinger equations in one space dimension, Diff. Integral Eqs., 7 (1994), 453-461.

[8] - Global, small radially symmetric solutions to nonlinear Schrödinger equations and a gauge transformation, Diff. Integral Eqs., 8 (1995), 1061-1072.

[9] Hörmander, L., The analysis of linear partial differential operators III, Springer-Verlag, Berlin, 1985.

[10] Katayama, S. and Tsutsumi Y., Global existence of solutions for nonlinear Schrödinger equations in one space dimension, Comm. P. D. E., 19(1994), 1971-1997.

[11] Kenig, C. E., Ponce, G. and Vega, L., Small solutions to nonlinear Schrödinger equations, Ann. I. H. P. Non. Lin., 10 (1993), 255-288.

[12] Klainerman. S., Global existence for nonlinear wave equations, Comm. Pure Appl. Math., 33 (1980), 43-101.

[13] Klainerman, S., and Ponce, G., Global, small amplitude solutions to nonlinear evolution equations, Comm. Pure Appl. Math., 36(1983), 133-141.

[14] Kumano-go, H., Pseudo-differential operators, MIT press, Cambridge, 1981.

[15] Mizohata, S., On the Cauchy problem, Academic Press, New York, 1985.

[16] Moser, J., A rapidly convergent interaction method and non-linear partial differential equations-I, Ann. Sc. Norm. Sup. Pisa (3), 20(1966), 265-315.

[17] Nirenberg, L., On elliptic partial differential equations, Ann. Sc. Norm. Sup. Pisa (3), 13 (1959), 115-162.

[18] Ozawa, T., Remarks on quadratic nonlinear Schrödinger equations, Funkcial. Ekvac., 38 (1995), 217-232.

[19] Shatah, J., Global existence of small solutions to nonlinear evolution equations, J. Diff. Eqs., 46 (1982), 409-425.

[20] Soyeur, A., The Cauchy problem for the Ishimori equations, J. Funct. Anal., 105 (1992), 233-255. 
